\title{
Human papillomavirus infection as a prognostic marker for lung adenocarcinoma: a systematic review and meta-analysis
}

\author{
Lanwei Guo ${ }^{1}$, Shuzheng Liu ${ }^{1}$, Shaokai Zhang ${ }^{1}$, Qiong Chen ${ }^{1}$, Meng Zhang ${ }^{1}$, Peiliang \\ Quan ${ }^{1}$ and Xibin Sun ${ }^{1}$ \\ ${ }^{1}$ Department of Cancer Epidemiology, Henan Office for Cancer Control and Research, The Affiliated Cancer Hospital of \\ Zhengzhou University, Henan Cancer Hospital, Zhengzhou, China
}

Correspondence to: XiBin Sun, email: xbsun21@sina.com

Keywords: HPV, lung cancer, prognosis, meta-analysis

Received: July 26, $2016 \quad$ Accepted: February 08, $2017 \quad$ Published: February 24, 2017

Copyright: Guo et al. This is an open-access article distributed under the terms of the Creative Commons Attribution License (CC-BY), which permits unrestricted use, distribution, and reproduction in any medium, provided the original author and source are credited.

\section{ABSTRACT}

\begin{abstract}
Although a number of studies have investigated the association between human papillomavirus (HPV) and lung cancer prognosis, the results remain inconsistent. We therefore conducted a meta-analysis of epidemiologic studies to address this issue. Searches of the MEDLINE and EMBASE electronic databases from their inception until June 30, 2016 yielded nine studies involving a total of 1,205 lung cancer cases that were used to conduct the meta-analysis. Study-specific risk estimates were pooled using a random-effects model. The pooled hazard ratio (HR) comparing HPV-positive to HPV-negative cancers 1.00 (95\% confidence interval (CI): 0.78-1.28) was not significantly correlated with overall survival. However, lung adenocarcinoma patients with HPV infections exhibited a survival benefit compared to those without HPV infection ( $\mathrm{HR}=0.69,95 \% \mathrm{CI}$ : 0.50-0.96). This meta-analysis suggests HPV infection is a prognostic marker in lung adenocarcinoma. To further elucidate the epidemiology and pathogenesis of HPV infections in lung cancer, future large prospective studies are encouraged to stratify survival analysis based on the pathological type and clinical stage of the cancer.
\end{abstract}

\section{INTRODUCTION}

Globally, lung cancer is the most common cancer overall for several decades, with an estimated 1.8 million new cases in 2012 and also the most common cause of death from cancer with an estimated 1.6 million deaths [1]. The number of lung cancer deaths are expected to reach 2.9 million by the year 2035 [1].

Although cigarette smoking is a predominant factor for lung cancer incidence, $25 \%$ of lung cancer patients are nonsmokers. There are many other potential risk factors for lung cancer, such as occupational or environmental exposure to radon and asbestos, certain metals and air pollution, as well as infectious diseases $[2,3]$. As reported, the high-risk oncogenic human papillomavirus (HPV) prevalence was $89.7 \%$ in cervical cancer [4], 29.5\% in head and neck cancer [5] and $22.0 \%$ in lung cancer [6]. And HPV has been identified as a causal agent in a variety of human carcinomas, including cervix cancer and head and neck cancer [7-9]. It was not clear whether HPV was implicated in lung carcinogenesis.

One study reported that HPV-positive cervical cancer patients who receiving radiation therapy had significantly better survival [10]. Some retrospective clinical studies consistently proved that HPV-positive head and neck squamous cell carcinoma (HNSCC) patients had a better prognosis than those HPV-negative [11-14]. Lungs can be infected just like the oral cavity, tonsils, and pharynx, it is supposed that the histological similarities between the head and neck squamous epithelia and lung suggest a similar association and clinical characteristics. Although the prognostic value of the HPV status has been investigated in patients with lung cancer previously, the results have often been controversial.

Therefore, this systematic review and meta-analysis was conducted to clarify the association between HPV infection and overall survival (OS) in lung cancer patients. 


\section{RESULTS}

\section{Literature search}

As shown in Figure 1, a total of 73 citations were generated, of which 32 were considered of potential value after screening titles and abstracts, and 19 articles were remained and retrieved for detailed evaluation after reading the full text. Eleven of these 19 articles were subsequently excluded for various reasons, including two reviews, eight that did not provide $H R \mathrm{~s}$ and one that was not based on lung cancer. Finally, eight eligible articles were included in this systematic review and meta-analysis [15-22].

\section{Characteristics of the selected articles}

Individual characteristics of the included 8 articles are summarized in Table 1. All included articles, satisfying all the five points proposed by MOOSE, had a very high quality. They were published from 2000 to 2014 and included a total of 1,205 lung cancer cases. Among these articles, five studies were conducted in China [16-18, 21, 22], two in Japan [19, 20] and one in Russia [15]. Of all the selected articles, three evaluated $H R \mathrm{~s}$ [15, 16, 21], while in the other five articles [17-20,22], HRs were absent, and needed to be calculated from the survival curves. Three articles did not give accurate data for follow-up [16, 19, 20]. The median follow-up period of other five articles ranged from 12.6 to 59.3 months.

\section{Results of the meta-analysis}

One included article [20] reported the HRs of lung adenocarcinoma and lung squamous cell carcinoma, respectively, and hence it was treated as two studies. Among the nine studies included, five [17-21] showed negative association comparing HPV-positive to HPV-negative cancers, one [20] showed statistical significance and the other four $[15,16,20,22]$ showed positive associations without statistical significance. The heterogeneity test indicated moderate degree of heterogeneity among included studies (Q-test $P_{\text {heterogeneity }}$ $\left.=0.038, I^{2}=51.0 \%\right)$ and therefore, random effects model was used to obtain the pooled $H R$. The pooled $H R$ of HPVpositive to HPV-negative cancers was 1.00 (95\% CI: 0.781.28 ) according to the nine individual effect estimates, suggesting no significant correlation with OS (Figure 2).

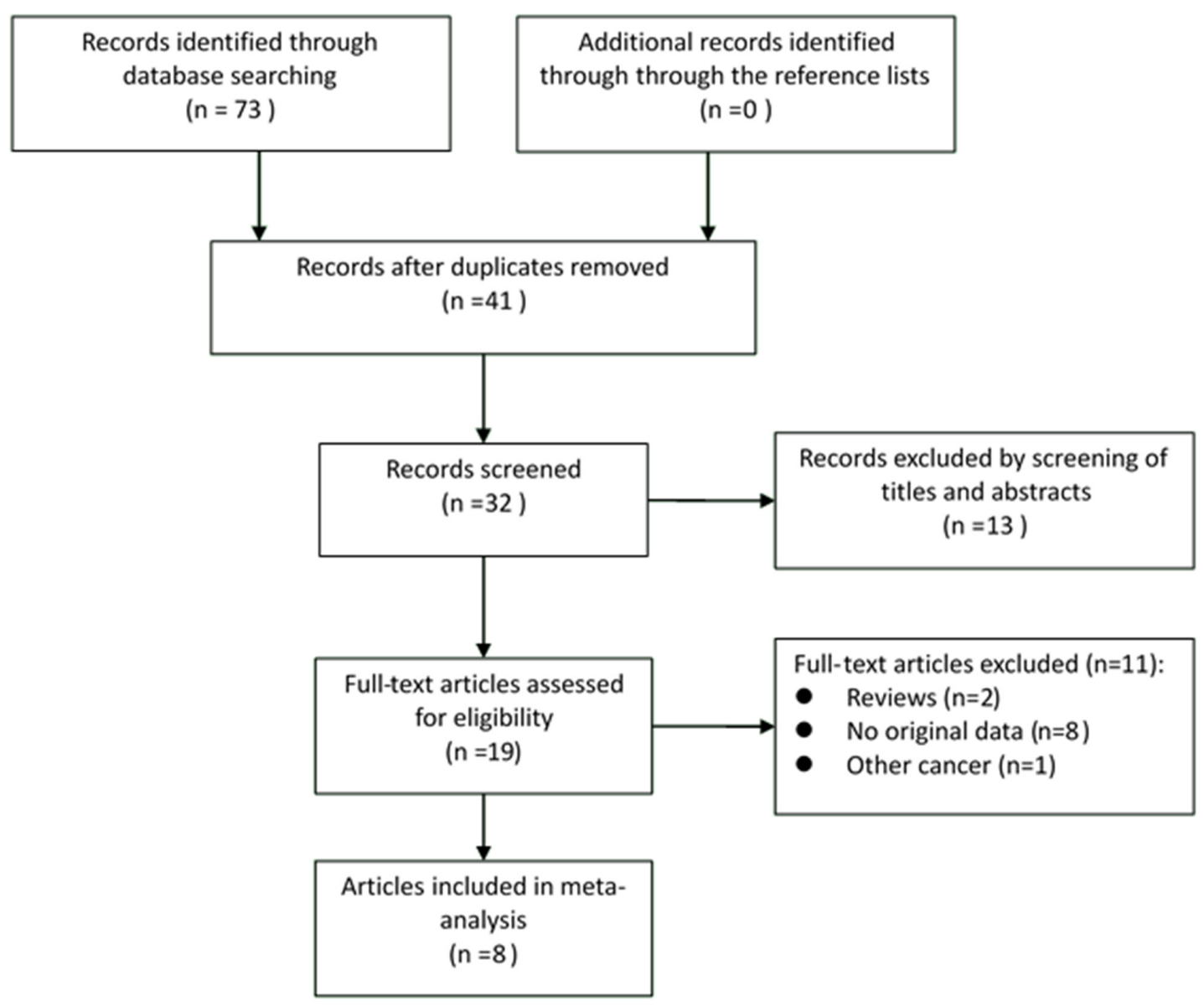

Figure 1: Flow diagram of systematic literature search. 
Table 1: Characteristics of the included studies

\begin{tabular}{|c|c|c|c|c|c|c|c|c|c|c|c|c|}
\hline First author & Year & $\begin{array}{c}\text { Year of } \\
\text { recruitment }\end{array}$ & Race & Stage & $\begin{array}{c}\text { Pathological } \\
\text { type }\end{array}$ & $\begin{array}{c}\text { No. of } \\
\text { Patients }\end{array}$ & $\begin{array}{c}\text { HPV + ve } \\
N(\%)\end{array}$ & Age, $y$ & $\begin{array}{c}\text { Genotype } \\
\text { (s) }\end{array}$ & $\begin{array}{c}\text { DNA } \\
\text { method }\end{array}$ & $\begin{array}{l}\text { Median } \\
\text { follow-up } \\
\text { period } \\
\text { (months) }\end{array}$ & $\begin{array}{c}\text { Hazard } \\
\text { ratio }\end{array}$ \\
\hline Iwamasa & 2000 & 1993-1995 & Asian & I-II & LSCC & 41 & $25(61.0)$ & $69.8(+) / 70.8(-)$ & $6,11,16,18$ & PCR & NA & $\mathrm{SC}$ \\
\hline Miyagi & 2001 & 1995-1997 & Asian & I-II & $\mathrm{LA}+\mathrm{LSCC}$ & 120 & $41(34.2)$ & $\begin{array}{c}\text { LA: } 67.3(+) / 66.9(-) \\
\text { LSCC: } \\
66.3(+) / 69.7(-)\end{array}$ & $6,11,16,18$ & PCR & NA & $\mathrm{SC}$ \\
\hline Hsu & 2009 & 2000-2006 & Asian & I & NSCLC & 171 & $17(9.9)$ & $65.2(37-83)$ & 16,18 & IMC & 56.4 & $\mathrm{SC}$ \\
\hline $\mathrm{Wu}$ & 2012 & 1998-2014 & Asian & I-III & NSCLC & 165 & $74(44.8)$ & NA & 16,18 & PCR & 59.3 & $\mathrm{SC}$ \\
\hline Chen & 2013 & $2002-2007$ & Asian & I-III & LC & 319 & $91(28.5)$ & NA & 16,18 & PCR & 57.6 & $\mathrm{SC}$ \\
\hline Anantharaman & 2014 & 2007-2010 & Caucasian & NA & LC & 62 & $15(24.2)$ & 62.1 & 21 types* & PCR & 43.44 & Report \\
\hline Chen & 2014 & 1993-2014 & Asian & NA & NSCLC & 117 & $62(53.0)$ & NA & 16,18 & PCR & NA & Report \\
\hline Wang & 2014 & 2003-2011 & Asian & I-IV & LA & 210 & $74(35.2)$ & 69.5 & 16,18 & PCR & 12.6 & Report \\
\hline
\end{tabular}

Abbreviations: HPV + ve, human papillomavirus positive; LSCC, lung squamous cell carcinoma; LA, lung adenocarcinoma; NSCLC, non-small cell lung cancer; LC, lung cancer; PCR, polymerase chain reaction; IMC, immunohistochemistry; SC, survival curve; NA, not available.

* Including 19 high-risk (HPV-16, 18, 26, 31, 33, 35, 39, 45, 51, 52, 53, 56, 58, 59, 66, 68a, 68b, 70, 73, 82) and 2 low-risk types (HPV-6, 11).

\section{Subgroup analyses}

Table 2 presents detailed results of subgroup analyses. The associations of HPV status and OS in lung cancer patients did not differ by study type, race, number of patients, detection method, HPV type, max follow-up time, case diagnosis method and hazard ratio. HPV status was significantly associated with improved OS for lung adenocarcinoma patients ( $H R=0.69,95 \% C I$ : $0.50-0.96)$, but was not significantly associated with OS for lung squamous cell carcinoma patients $(H R=0.50,95 \% C I$ : $0.23-1.12)$, non-small cell lung cancer patients $(H R=0.92$, $95 \% C I$ : $0.65-1.31)$ and lung cancer patients $(H R=1.17$, 95\% CI: 0.68-2.03). When cancer cases were stratified by treatment method, HPV status was not significantly associated with improved OS for surgery only ( $H R=0.97$, 95\% CI: 0.73-1.28), but was significantly associated with better OS for other treatment methods ( $H R=0.72,95 \% C I$ : $0.58-0.89)$. In short, the estimated heterogeneity remained for the included studies although it decreased to some extent.

\section{Influence analysis of individual studies}

To address the potential bias due to the quality of included studies, we performed the sensitivity analysis by calculating pooled $H R$ s again by omitting one study at a time. And related results were showed in Figure 3. The pooled HRs comparing HPV-positive to HPV-negative cancers ranged from 0.94 (95\% CI: 0.74$1.21)$ to 1.10 (95\% CI: $0.87-1.39$ ), which indicated that each single study didn't influence the stability of pooled $H R$ estimate.

\section{Publication bias}

Both the non-significant $P$ values of Begg's test (1.000), Eegg's test (0.760), and the near-symmetric funnel plot demonstrated that there was no publication bias (Figure 4).

\section{DISCUSSION}

As we know, this systematic review has its first-ever try on investigating overall survival of HPV-related lung cancers. The pooled effect estimates showed that lung adenocarcinoma patients with HPV infections survived better than those without.

The association between HPV infection and the occurrence of lung cancer was firstly reported by Syrjänen in 1979 [23], and several studies further explored impact of HPV infection on lung cancer prognosis. However, the conclusions of these studies were inconsistent. One previous study reported that HPV-positive HNSCCs was associated with a $54 \%$ reduction in overall mortality, in comparison to HPV-unrelated HNSCCs [24]. However, no association was found between HPV status and lung cancer prognosis in this meta-analysis. Among the included studies, only Wang et al. [21] demonstrated that patients with HPV-positive lung adenocarcinoma had a superior prognosis than patients with HPV-negative ones, with a $32 \%$ reduction in overall mortality. However, it is unclear about the biologic basis for the improved survival among the HPV positive patients and further study is needed.

Although limitations existed due to observational nature, it deserved to note some findings from subgroup- 
analysis. HPV infection was associated with improved survival for lung adenocarcinoma patients, but was not associated with improved survival for lung squamous cell carcinoma patients. Besides, when stratified by treatment method, HPV status was not significantly associated with improved OS for surgery only patients, but significantly associated with improved OS for patients with other treatment methods, such as chemotherapy, radiotherapy, tyrosine kinase inhibitors and multiple therapies. Further studies on the difference in survival between HPV status and different pathological types of lung cancer with different treatment methods were encouraged.

Clinical stage at diagnosis is the most important prognostic factor for lung cancer [25]. It's also a pre-requisite for identifying lung cancer patients as candidates for chemo-radiotherapy prior to surgery. For lung adenocarcinoma, one included study [21] showed HPV-positive stage I-IV patients having favorable survival, but HPV-positive patients stage I-II showed poorer survival in another study [20]. For LSCC, two studies $[19,20]$ showed HPV-positive stage I-II patients having favorable survival. For NSCLC (except lung adenocarcinoma and LSCC), one included study [18] showed HPV-positive stage I NSCLC patients having favorable survival, but HPV-positive patients stage I-III or I-IV showed poorer survival in two other studies [16,
22]. However, only three studies reported the adjusted $H R \mathrm{~s}$, one [16] of which reported the adjusted $H R \mathrm{~s}$ for clinical stage. The favorable prognosis for HPV-infected lung adenocarcinoma or NSCLC patients compared with HPV-non-infected patients could be due to different tumor stages in the patients. So, it is recommended to do detailed survival analysis by different clinical stages of lung cancer for future studies.

Obviously, this systematic review and meta-analysis has several strengths. First, it is the first time to explore survival differences in different HPV status among lung cancers to date, and this comprehensive review is the most methodologically robust. Second, rigorous inclusion/ exclusion criterion and advanced meta-analysis of $H R$ for survival were applied. Moreover, a variety of subgroup analyses were conducted, which means a minimized potential confounders. In addition, no publication bias and the robust results of sensitivity analysis indicated the reliability of our study.

Limitations of this meta-analysis should be considered. First, potential bias could not be completely excluded considering that different HPV DNA detection methods were used in the included studies, and the estimates of HPV infection might be influenced largely due to the difference of the sensitivity and accuracy of the detection methods. Second, though random-effects model meta-analysis was used whenever significant heterogeneity

\begin{tabular}{|c|c|c|}
\hline Study & & $\%$ \\
\hline ID & HR $(95 \% \mathrm{Cl})$ & Weight \\
\hline
\end{tabular}

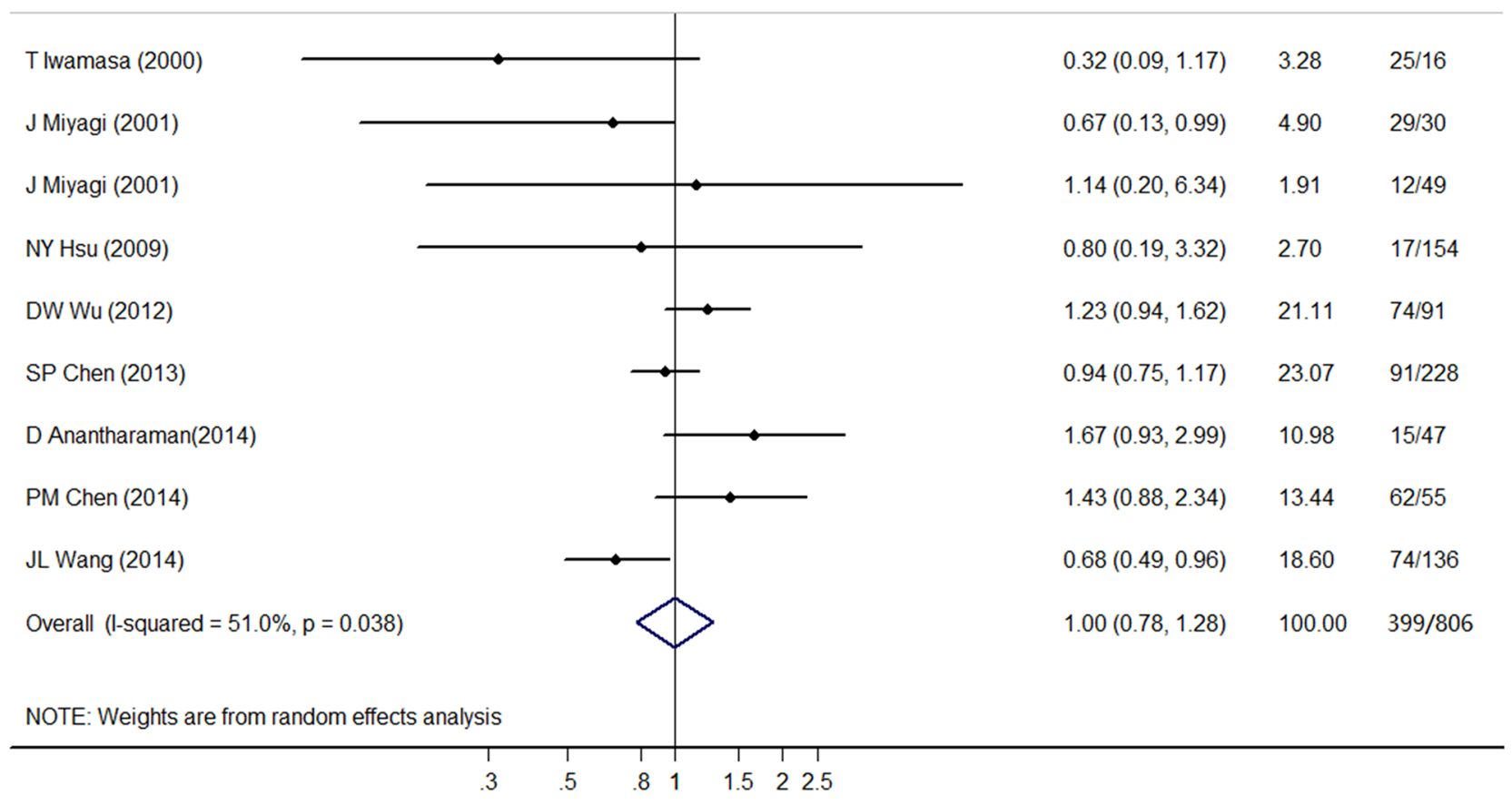

Figure 2: Forest plot comparing HPV-positive to HPV-negative lung cancer patients and overall survival. 
Table 2: Results of subgroup analyses

\begin{tabular}{|c|c|c|c|c|}
\hline \multirow[t]{2}{*}{ Group } & \multirow[t]{2}{*}{ No. of study } & \multirow[t]{2}{*}{$H R(95 \% C I)$} & \multicolumn{2}{|c|}{ Heterogeneity test } \\
\hline & & & $P$ for $Q$ test & $I^{2}, \% \dagger$ \\
\hline All & 9 & $1.00(0.78-1.28)$ & 0.038 & 51.0 \\
\hline \multicolumn{5}{|l|}{ Study type } \\
\hline Prospective & 8 & $0.94(0.74-1.21)$ & 0.068 & 46.9 \\
\hline \multicolumn{5}{|l|}{ Race } \\
\hline Asian & 8 & $0.94(0.74-1.21)$ & 0.068 & 46.9 \\
\hline \multicolumn{5}{|l|}{ Number of patients } \\
\hline$<100$ & 4 & $0.86(0.43-1.72)$ & 0.134 & 46.2 \\
\hline$\geq 100$ & 5 & $1.01(0.76-1.34)$ & 0.031 & 62.3 \\
\hline \multicolumn{5}{|l|}{ Pathological type } \\
\hline $\mathrm{LC}$ & 2 & $1.17(0.68-2.03)$ & 0.071 & 69.2 \\
\hline NSCLC & 7 & $0.92(0.65-1.31)$ & 0.042 & 54.1 \\
\hline LSCC & 2 & $0.50(0.23-1.12)$ & 0.376 & 0.0 \\
\hline LA & 2 & $0.69(0.50-0.96)$ & 0.565 & 0.0 \\
\hline \multicolumn{5}{|l|}{ Detection method } \\
\hline PCR & 8 & $1.01(0.78-1.31)$ & 0.023 & 56.9 \\
\hline \multicolumn{5}{|l|}{ HPV type } \\
\hline 16,18 & 6 & $1.06(0.82-1.38)$ & 0.028 & 60.2 \\
\hline HR/LR-HPV & 4 & $0.88(0.40-1.91)$ & 0.092 & 53.4 \\
\hline \multicolumn{5}{|l|}{ Max follow-up } \\
\hline$<5$ years & 2 & $0.77(0.32-1.84)$ & 0.603 & 0.0 \\
\hline$\geq 5$ years & 6 & $0.96(0.71-1.29)$ & 0.019 & 63.1 \\
\hline \multicolumn{5}{|l|}{ Treatment method } \\
\hline Surgery only & 6 & $0.97(0.73-1.28)$ & 0.308 & 16.4 \\
\hline Others* & 4 & $0.72(0.58-0.89)$ & 0.641 & 0.0 \\
\hline \multicolumn{5}{|c|}{ Case diagnosis method } \\
\hline Pathology reports & 8 & $1.10(0.87-1.39)$ & 0.174 & 31.8 \\
\hline \multicolumn{5}{|l|}{ Hazard ratio } \\
\hline Reported & 3 & $1.14(0.62-2.07)$ & 0.007 & 80.1 \\
\hline Estimated & 6 & $0.99(0.79-1.25)$ & 0.283 & 20.0 \\
\hline
\end{tabular}

Abbreviation: $H R$, hazard ratio; $C I$, confidence intervals; LC, lung cancer; NSCLC, non-small cell lung cancer; LSCC, lung squamous cell carcinoma; LA, lung adenocarcinoma; PCR, polymerase chain reaction; IMC, immunohistochemistry; HR, high-risk; LR, low risk;

$\dagger I^{2}$ is interpreted as the proportion of total variation across studies that are due to heterogeneity rather than chance;

*Including chemotherapy, radiotherapy, tyrosine kinase inhibitors and multiple therapies.

was noted and sensitivity with subgroup analyses were performed to figure out potential sources of heterogeneity, significant heterogeneity was observed. Third, only those articles published in English were included here, which may have introduced language bias as well. Finally, only one third included studies reported the adjusted $H R \mathrm{~s}$, which may exclude other potential prognostic factors such as smoking. 


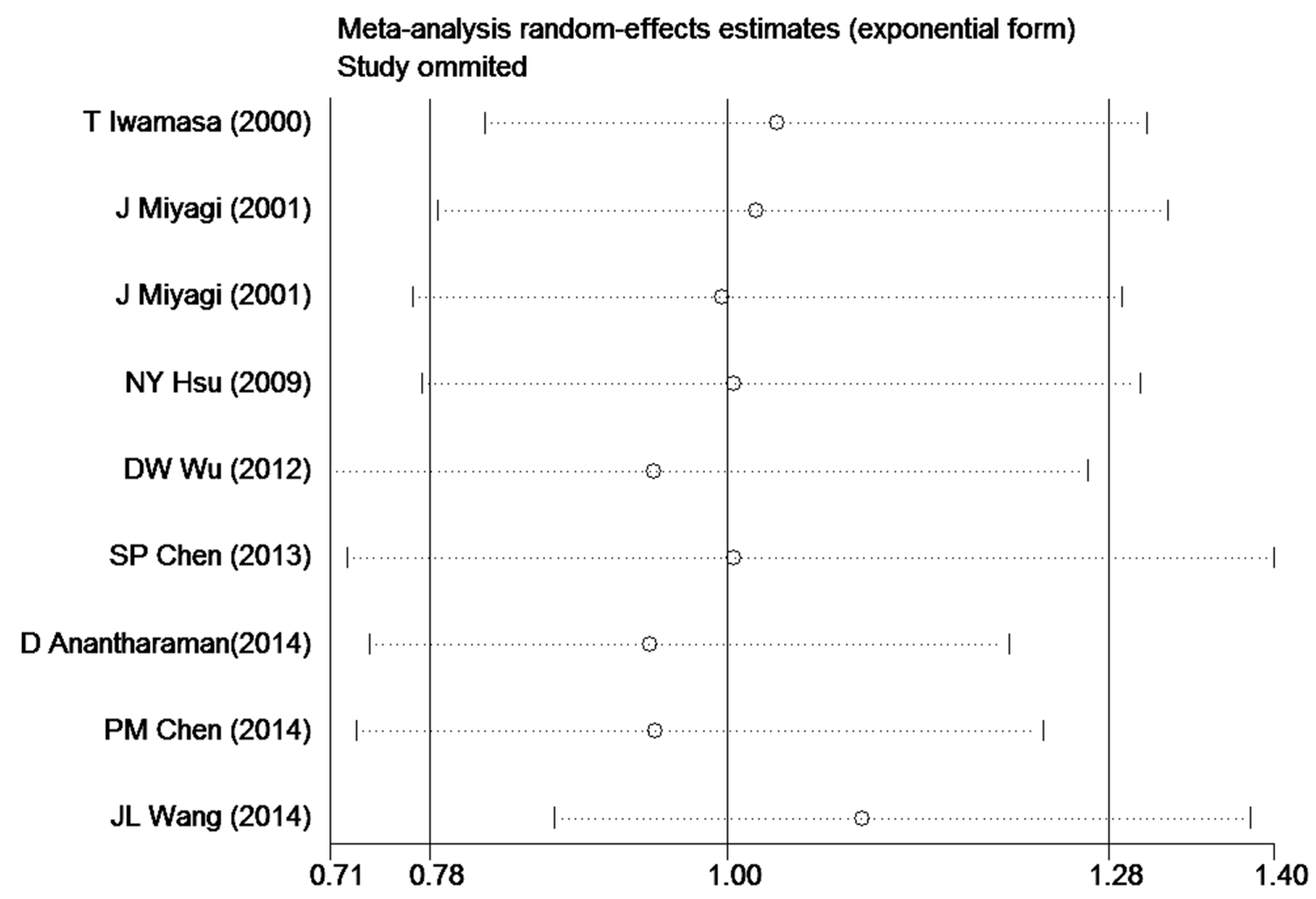

Figure 3: Influence analyses for omitting individual study on the summary $H R$ for overall survival.

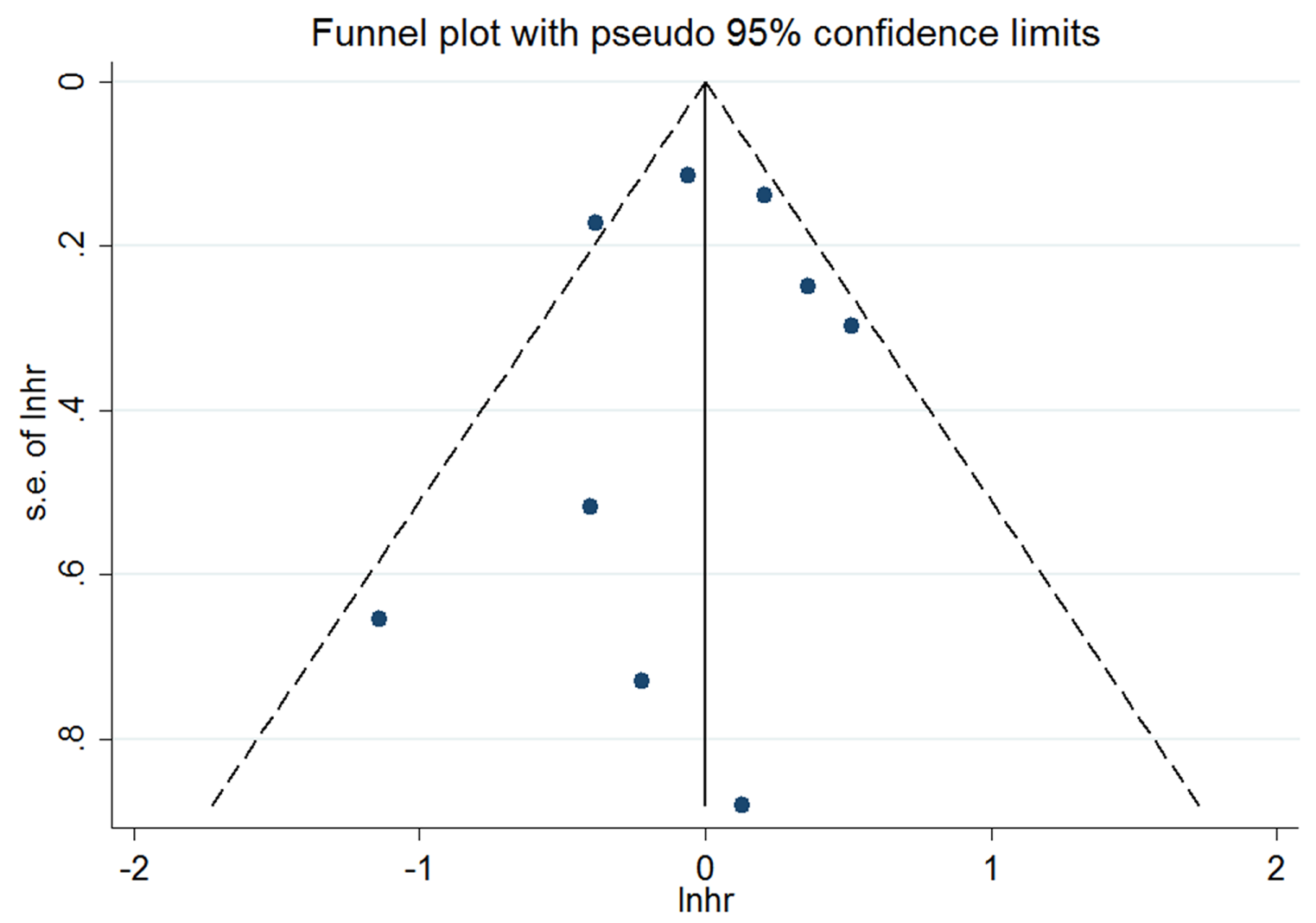

Figure 4: Funnel plots for publication bias of overall survival. 
In summary, no association was observed between HPV infection and lung cancer survival. However, HPV infection may be a prognostic marker in lung adenocarcinoma, which suggests that assessment of HPV infection in clinical practice might help to determine the relevant treatment regimen. To further elucidate the epidemiology and pathogenesis of HPV infections in lung cancer, future large prospective studies are encouraged to stratify survival analysis based on the pathological type and clinical stage of the cancer.

\section{MATERIALS AND METHODS}

\section{Literature search strategy}

A systematic search up to 30 Jun 2016 was conducted in MEDLINE (via PubMed) and Excerpta Medica database (EMBASE) to identify relevant articles. Search terms included "human papillomavirus OR HPV", "lung cancer OR lung neoplasms OR lung carcinoma" combined with "prognosis OR prognostic OR survival". Additional relevant references cited in retrieved articles were also evaluated.

\section{Inclusion and exclusion criteria}

All papers were reviewed by two authors (S.Z. and Q.C.) independently. Uncertainties and discrepancies were resolved by consensus after discussing with a senior researcher (P.Q.). All studies included in the final meta-analysis satisfied the following criteria: (a) patients were pathologically diagnosed as lung cancer; (b) lung cancer OS as the outcome of interest; (c) reported $H R$ estimates with their corresponding 95\% CI (or sufficient data to calculate of these effect measure), and (d) English articles. If the study was reported in duplication, the one published earlier or provided more detailed information was included. Review articles and editorials were included if they contained original data. Abstracts were excluded.

\section{Quality assessment}

According to a critical review checklist of the Dutch Cochrane Centre proposed by MOOSE, we strictly assessed the quality of all the studies included [26]. (i) clear definition of study population and origin of country; (ii) clear definition of study design; (iii) clear definition of outcome assessment; (iv) clear definition of HPV detection method and (v) sufficient period of follow-up. Otherwise, we would exclude the studies in order to ensure the quality of the meta-analysis.

\section{Data extraction}

Two of the authors (S.L. and S.Z.) performed the data extraction from each article and discrepancies were resolved by consensus. For studies meeting our inclusion criteria, a standardized data extraction form was used to extract the following data: the first author's name, year of publication, country of origin, study design, period of enrollment, the length of follow-up, characteristics of the studied population (sample size, age, stage of disease and treatment method), HPV detection methods, and $H R$ estimates for OS with corresponding 95\% CIs. When data for $H R$ was not available, we extracted the total numbers of observed deaths and the numbers of patients in each group to calculate $H R$ [27]. Data were extracted by Engauge Digitizer version 4.1 (http://digitizer. sourceforge.net/) from the graphical survival plots when data were only available as Kaplan-Meier curves [28], then the estimation of the $H R$ was performed by the described method [27].

\section{Statistical analysis}

The $H R$ with $95 \% C I$ was used to compute the pooled HPV infections and the OS in lung cancer patients. A random-effect model was used to pool the data, based on the DerSimonian and Laird method [29].

Cochrane $Q$ test $(P<0.10$ indicated a high level of statistical heterogeneity) and $I^{2}$ (values of $25 \%, 50 \%$ and $75 \%$ corresponding to low, moderate and high degrees of heterogeneity, respectively) was used to assess the heterogeneity between eligible studies, which test total variation across studies that was attributable to heterogeneity rather than to chance [30]. Subgroup analyses for HPV infections and the OS in lung cancer patients were subsequently carried out according to the study type, race, number of patients, pathological type, detection method, HPV type, max follow-up time, treatment method, case diagnosis method and hazard ratio. Sensitivity analysis was also conducted to assess the influence of each individual study on the strength and stability of the meta-analytic results. Each time, one study in the meta-analysis was excluded to show that study's impact on the combined effect size. Funnel plot and Begg adjusted rank correlation test for funnel plot asymmetry were performed to test any existing publication bias.

All statistical analyses were performed using STATA version 12 for Windows (StataCorp LP, College Station, TX, USA). A two-tailed $P<0.05$ was considered statistically significant.

\section{Author contributions}

All authors have made substantial contributions to the conception and design of the study. L.G. contributed to protocol design, search, data extraction, quality assessment, statistical analysis, and writing the report. S.L. and S.Z contributed to protocol design, search, data extraction, and writing the report. Q.C., M.Z. and P.Q. contributed to quality assessment, statistical analysis, and revision of the report. X.S. contributed to interpretation of data and revision of the report. All authors have seen and approved the final version. 


\section{CONFLICTS OF INTEREST}

The authors have declared that no competing interests exist.

\section{REFERENCES}

1. Ervik M, Lam M, Ferlay J, Mery L, Soerjomataram I, Bray F. Cancer Today. Lyon, France: International Agency for Research on Cancer. Cancer Today. 2016; Available from: http://gco.iarc.fr/ today, accessed [09/07/2016].

2. Spyratos D, Zarogoulidis P, Porpodis K, Tsakiridis K, Machairiotis N, Katsikogiannis N, Kougioumtzi I, Dryllis G, Kallianos A, Rapti A. Occupational exposure and lung cancer. Journal of thoracic disease. 2013; 5:S440-445.

3. Hajdu SI, Ali SZ. Discovery of human papillomavirus in carcinoma of the lung. Annals of clinical and laboratory science. 2008; 38:3-5.

4. de Sanjose S, Diaz M, Castellsague X, Clifford G, Bruni L, Munoz N, Bosch FX. Worldwide prevalence and genotype distribution of cervical human papillomavirus DNA in women with normal cytology: a meta-analysis. The Lancet Infectious diseases. 2007; 7:453-459.

5. Ndiaye C, Mena M, Alemany L, Arbyn M, Castellsague X, Laporte L, Bosch FX, de Sanjose S, Trottier H. HPV DNA, E6/E7 mRNA, and p16INK4a detection in head and neck cancers: a systematic review and meta-analysis. The Lancet Oncology. 2014; 15:1319-1331.

6. Syrjanen K. Detection of human papillomavirus in lung cancer: systematic review and meta-analysis. Anticancer research. 2012; 32:3235-3250.

7. Ciapponi A, Bardach A, Glujovsky D, Gibbons L, Picconi MA. Type-specific HPV prevalence in cervical cancer and high-grade lesions in Latin America and the Caribbean: systematic review and meta-analysis. PloS one. 2011; 6:e25493.

8. Kreimer AR, Clifford GM, Boyle P, Franceschi S. Human papillomavirus types in head and neck squamous cell carcinomas worldwide: a systematic review. Cancer epidemiology, biomarkers \& prevention. 2005; 14:467-475.

9. Stelzer MK, Pitot HC, Liem A, Schweizer J, Mahoney C, Lambert PF. A mouse model for human anal cancer. Cancer prevention research. 2010; 3:1534-1541.

10. Harima Y, Sawada S, Nagata K, Sougawa M, Ohnishi T. Human papilloma virus (HPV) DNA associated with prognosis of cervical cancer after radiotherapy. International journal of radiation oncology, biology, physics. 2002; 52:1345-1351.

11. Reimers N, Kasper HU, Weissenborn SJ, Stutzer H, Preuss SF, Hoffmann TK, Speel EJ, Dienes HP, Pfister HJ, Guntinas-Lichius O, Klussmann JP. Combined analysis of HPV-DNA, p16 and EGFR expression to predict prognosis in oropharyngeal cancer. International journal of cancer. 2007; 120:1731-1738.
12. Syrjanen S. HPV infections and tonsillar carcinoma. Journal of clinical pathology. 2004; 57:449-455.

13. Kong CS, Narasimhan B, Cao H, Kwok S, Erickson JP, Koong A, Pourmand N, Le QT. The relationship between human papillomavirus status and other molecular prognostic markers in head and neck squamous cell carcinomas. International journal of radiation oncology, biology, physics. 2009; 74:553-561.

14. Weinberger PM, Yu Z, Haffty BG, Kowalski D, Harigopal M, Brandsma J, Sasaki C, Joe J, Camp RL, Rimm DL, Psyrri A. Molecular classification identifies a subset of human papillomavirus--associated oropharyngeal cancers with favorable prognosis. Journal of clinical oncology. 2006; 24:736-747.

15. Anantharaman D, Gheit T, Waterboer T, Halec G, Carreira C, Abedi-Ardekani B, McKay-Chopin S, Zaridze D, Mukeria A, Szeszenia-Dabrowska N, Lissowska J, Mates D, Janout V, et al. No causal association identified for human papillomavirus infections in lung cancer. Cancer research. 2014; 74:3525-3534.

16. Chen PM, Cheng YW, Wang YC, Wu TC, Chen CY, Lee H. Up-regulation of FOXM1 by E6 oncoprotein through the MZF1/NKX2-1 axis is required for human papillomavirus-associated tumorigenesis. Neoplasia. 2014; 16:961-971.

17. Chen SP, Hsu NY, Wu JY, Chen CY, Chou MC, Lee H, Cheng YW. Association of p53 codon 72 genotypes and clinical outcome in human papillomavirus-infected lung cancer patients. The Annals of thoracic surgery. 2013; 95:1196-1203.

18. Hsu NY, Cheng YW, Chan IP, Ho HC, Chen CY, Hsu CP, Lin MH, Chou MC. Association between expression of human papillomavirus 16/18 E6 oncoprotein and survival in patients with stage I non-small cell lung cancer. Oncology reports. 2009; 21:81-87.

19. Iwamasa T, Miyagi J, Tsuhako K, Kinjo T, Kamada Y, Hirayasu T, Genka K. Prognostic implication of human papillomavirus infection in squamous cell carcinoma of the lung. Pathology, research and practice. 2000; 196:209-218.

20. Miyagi J, Kinjo $T$, Tsuhako $K$, Higa $M$, Iwamasa $T$, Kamada Y, Hirayasu T. Extremely high Langerhans cell infiltration contributes to the favourable prognosis of HPVinfected squamous cell carcinoma and adenocarcinoma of the lung. Histopathology. 2001; 38:355-367.

21. Wang JL, Fang CL, Wang M, Yu MC, Bai KJ, Lu PC, Liu HE. Human papillomavirus infections as a marker to predict overall survival in lung adenocarcinoma. International journal of cancer. 2014; 134:65-71.

22. Wu DW, Tsai LH, Chen PM, Lee MC, Wang L, Chen CY, Cheng YW, Lee H. Loss of TIMP-3 promotes tumor invasion via elevated IL-6 production and predicts poor survival and relapse in HPV-infected non-small cell lung cancer. The American journal of pathology. 2012; 181:1796-1806. 
23. Syrjanen KJ. Condylomatous changes in neoplastic bronchial epithelium. Report of a case. Respiration. 1979; 38:299-304.

24. O'Rorke MA, Ellison MV, Murray LJ, Moran M, James J, Anderson LA. Human papillomavirus related head and neck cancer survival: a systematic review and meta-analysis. Oral oncology. 2012; 48:1191-1201.

25. Didkowska J, Wojciechowska U, Manczuk M, Lobaszewski J. Lung cancer epidemiology: contemporary and future challenges worldwide. Annals of translational medicine. 2016; 4:150.

26. Stroup DF, Berlin JA, Morton SC, Olkin I, Williamson GD, Rennie D, Moher D, Becker BJ, Sipe TA, Thacker SB. Meta-analysis of observational studies in epidemiology: a proposal for reporting. Meta-analysis Of Observational
Studies in Epidemiology (MOOSE) group. JAMA. 2000; 283:2008-2012.

27. Parmar MK, Torri V, Stewart L. Extracting summary statistics to perform meta-analyses of the published literature for survival endpoints. Statistics in medicine. 1998; 17:2815-2834.

28. Tierney JF, Stewart LA, Ghersi D, Burdett S, Sydes MR. Practical methods for incorporating summary time-to-event data into meta-analysis. Trials. 2007; 8:16.

29. DerSimonian R, Kacker R. Random-effects model for metaanalysis of clinical trials: an update. Contemporary clinical trials. 2007; 28:105-114.

30. Higgins JPT, Thompson SG. Quantifying heterogeneity in a meta-analysis. Statistics in medicine. 2002; 21:1539-1558. 